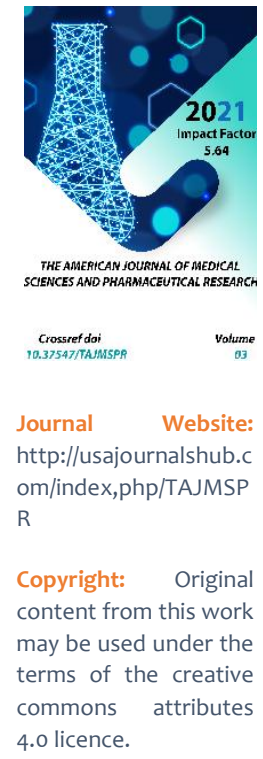

\title{
Morphological Changes In Bone Tissue In Chronic Osteomyelitis On The Background Of Application Of Plate Concentrate
}

\author{
Nurulloev Sukhrob Ozodovich \\ Assistant Of Department Of Traumathology And Pediatric Surgery, Bukhara State Medical \\ Institute, Uzbekistan
}

Mirzamuradov Habib Halimovich

Assistant Of Department Of Traumathology And Pediatric Surgery, Bukhara State Medical Institute, Uzbekistan

\section{ABSTRACT}

The method of laser osteoperforation developed experimentally was approved in 142 patients with chronic osteomyelitis of different forms. A diode laser was used in which wavelength was $970 \mathrm{~nm}$ and power $-30 \mathrm{~W}$. Laser osteoperforation was performed transcutaneously in a contact way with a quartz light guide of 0, $4 \mathrm{~mm}$ diameter in the projection of bone destruction foci with formation of 2-20 holes. Normalization of temperature and blood indices as well as pain decrease, reduce of soft tissue tension and edema was observed in all the patients in the immediate days after surgery. As a rule, fistulae closed by 4-10 day. In case of chronic osteomyelitis persistent remission is noted during 2-5 years by the objective data (roentgenography, flowmetry, densitometry). Thus, the experience of laser osteoperforation clinical use demonstrates its high effectiveness in treatment of osteomyelitis different forms. The method is of little invasiveness and it allows to reduce the periods of treatment and rehabilitation to a great extent.

\section{KEYWORDS}

Diode laser, osteomyelitis, morphology, roentgenography, densitometry, flowmetry. 


\section{INTRODUCTION}

According to the WHO, osteomyelitis accounts for up to $6.5-7 \%$ in the general structure of diseases of the support and movement organs. It often develops after open fractures of tubular bones (10-22\%), surgical treatment of closed fractures (2-7\%), gunshot fractures (90\%). In $18-24 \%$ of children with acute hematogenous osteomyelitis, a transition to the chronic stage is noted, and almost $10 \%$ develop disability. Until now, a unified surgical tactic has not been developed, which leads to many methods of surgical treatment $[1,5$, 6].The urgency of the problem of treating chronic osteomyelitis (CO) is determined by the significant prevalence of the disease due to the steady increase in injuries, as well as the severity and duration of the pathological process, the difficulties of prevention and treatment of this disease [1-4]. The pathomorphological basis of chronic osteomyelitis is a complex of ischemic, infectious-inflammatory and reparative changes in the bone and surrounding soft tissues. These structural and functional changes are determined by the characteristics of the causative agents of the infectious process, the nature and severity of inflammatory and proliferative processes in the affected area.

The aim of this work was to establish morphological changes in bone and surrounding tissues in chronic experimental osteomyelitis against the background of selective and combined regional use of platelet concentrate.

\section{MATERIALS AND METHODS}

The study was carried out on 28 white outbred rats, in which, under anesthesia with Zolityl-100 at a dosage of $8 \mu / \mathrm{kg}$ in aseptic conditions, CT was modeled by open osteotomy in the area of the distal metaepiphysis of the femur, followed by infection of the bone injury site with a culture of pathogenic Staphylococcus aureus (108 microbial bodies).

After the simulation of $\mathrm{CT}$ ( 31 days from the moment of introducing the pathogenic culture), surgical debridement of the focus was performed in all experimental groups, which consisted of removing sequesters, cleansing the walls of the bone cavity until the appearance of "blood dew". In the 1st experimental group, the treatment consisted of blasting the damaged area using $0.9 \%$ sodium chloride solution. In the 2nd experimental group, a platelet concentrate (TC) with a platelet concentration of $1 \mathrm{mln} / \mu \mathrm{L}$ was used for treatment. In the 3 rd test group, the animals received a combined treatment, including the implementation of jet sanitation and the introduction of TC. In the control group, no treatment was carried out. The animals were taken out of the experiment under anesthesia for 14 days. Fragments of tissues in the CT zone were subjected to histological processing - they were fixed in 10\% neutral formalin, decalcified, paraffin sections were made, stained with hematoxylin and eosin and according to Van Gieson. Based on the results of the morphological study, the nature and severity of inflammation, the presence of foci of destruction were assessed, and reparative changes in the bone were also studied.

\section{RESULTS AND ITS DISCUSSION}

In all experimental groups, structural and functional changes were expressed in the presence of necrotic areas of bone tissue, but, in addition to destructive changes along the periphery of the osteomyelitis focus, there was a proliferative reaction in the form of the formation of granulation tissue with a numerous cellular component, represented by fibroblasts, osteoblasts. The outer sections of the granulation tissue are surrounded by a fibrous membrane. As a result of the 
inflammatory reaction and the accompanying acidosis, vascular obstruction occurred. In conditions of impaired blood supply, bone trabeculae die and become fragmented. The most significant areas of necrotic bone with the formation of sequesters were observed in the control group. In the 1st experimental group, the necrotic areas of the bone tissue were small, but some of them were freely located in the abscess cavity. In the 2nd test group, there was an accumulation of pus under the periosteum, in the marginal zone of damage, the activity of osteoblasts was increased, which indicated active processes of bone formation, there were dense sequesters, the formation of which can be explained by

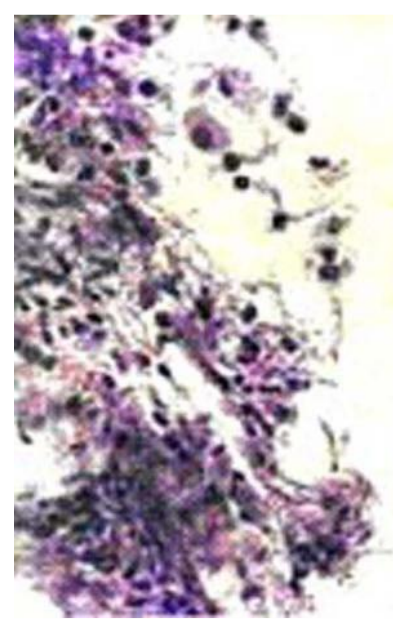

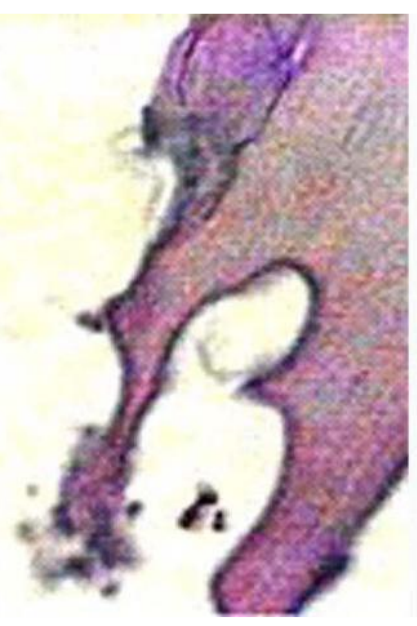

A increased reparative processes in bone tissue associated with an increase in the activity of osteoblasts on the surface. old trabeculae. In the 3 rd test group, elements of an inflammatory-destructive focus were determined in the study area: necrotic bone in the center of the focus contained fibrouspurulent inflammatory infiltrate in the vascular canals and interbeam spaces; the process of sequestration was observed with the formation of resorption cavities filled with granulation tissue. In some cases, bone fragments were fused with fibrocartilaginous tissue of a heterogeneous degree of maturity, in which macrophage-plasmacytic infiltrates were encountered.

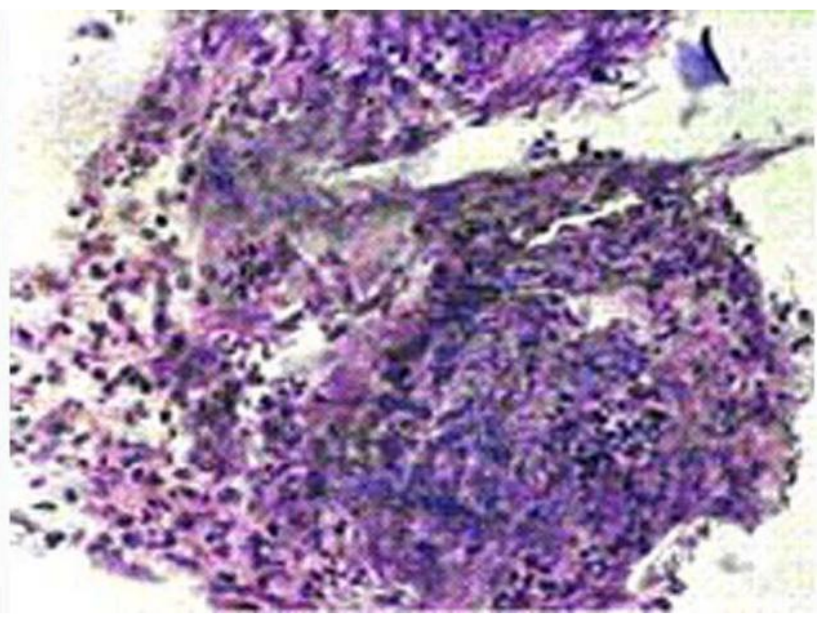




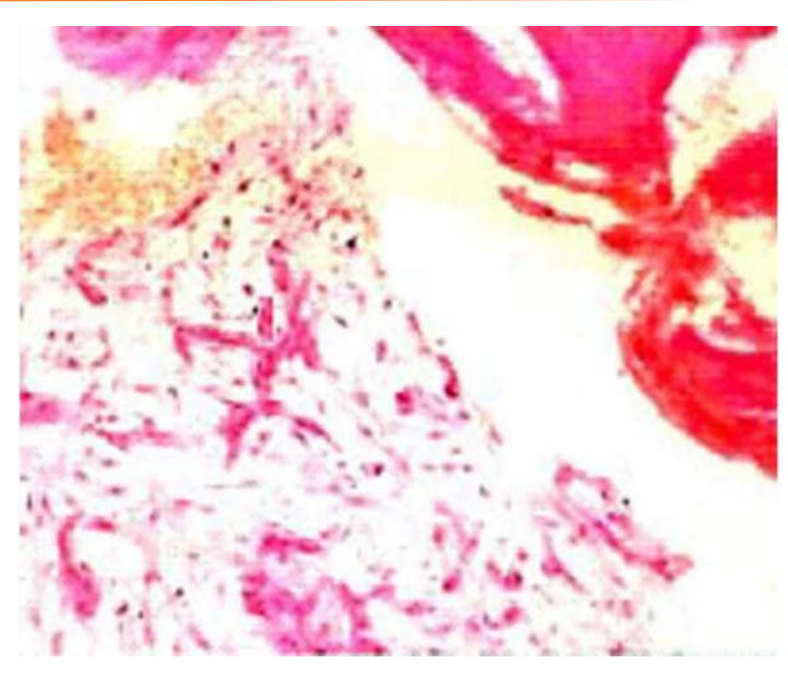

C

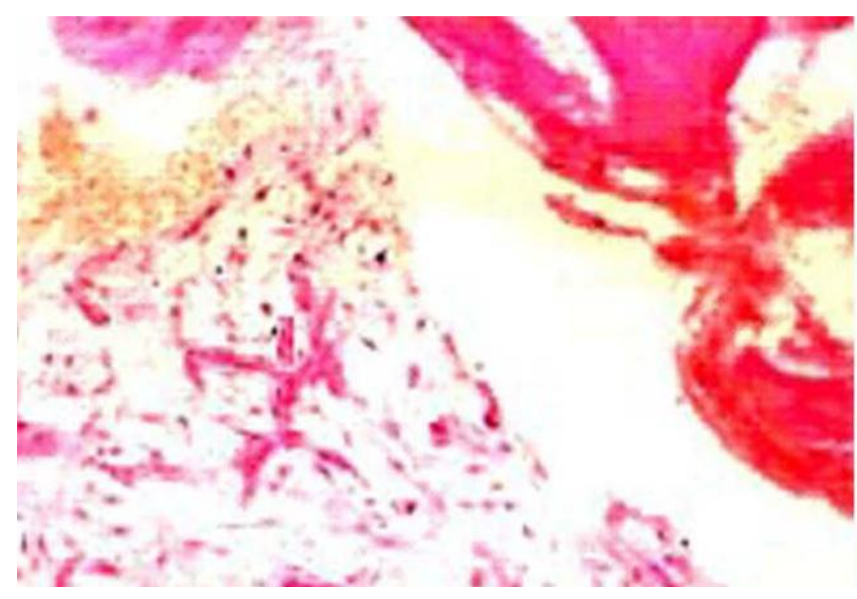

E

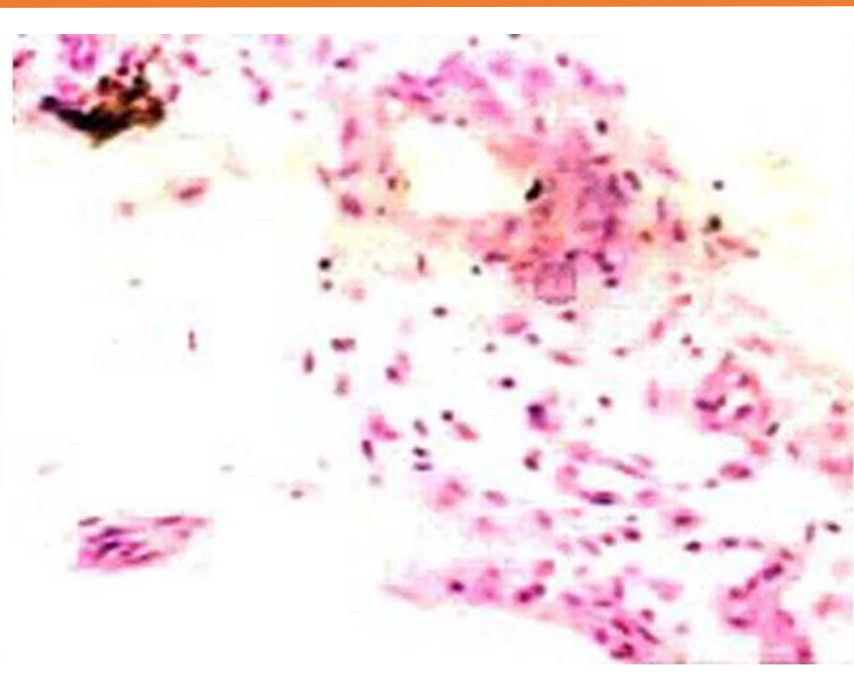

D

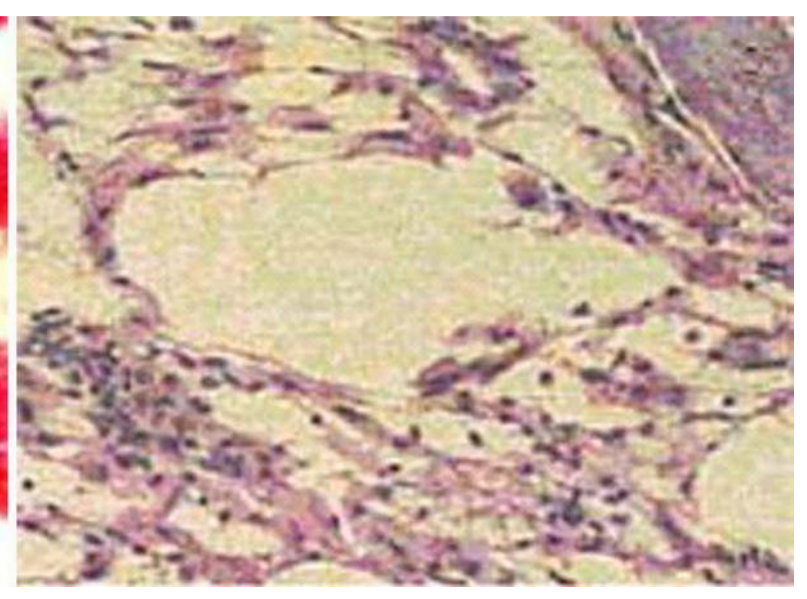

$\mathrm{F}$

Fig. 1. Data of morphological studies of bone tissue in the compared groups. Hematoxylin and eosin staining, magnification 400: $a 2$ weeks after laser exposure; $b 2$ weeks after traditional treatment; $c 1$ month after laser exposure; $d 1$ month after traditional treatment; $e 6$ months after laser exposure; e 6 months after traditional treatment

Compared with other groups, there was a predominance of bone regenerates, which are elements of the bone membrane of the osteomyelitic focus, built from immature bone tissue. The outer surface of the capsule that delimits the CS focus is represented by dense fibrous connective tissue containing fibroblasts and collagen fibers of various thicknesses. In contrast to other experimental groups, the combined use of $M C$ led to a change in the ratio of the cellular-fibrous 
component towards an increase in collagen fibers, the number of vessels was reduced.

\section{CONCLUSION}

The data obtained indicate morphofunctional rearrangements within the $\mathrm{CO}$ zone during selective and combined treatment with the use of MC. Stimulation of proliferative processes with this method of regional influence is most effective after blasting of the osteomyelitis focus, as evidenced by the increased activity of osteoblasts, leading to the formation of bone regenerates filling the bone defect.

\section{REFERENCES}

1. Belyaeva, A.A. Treatment of chronic posttraumatic osteomyelitis of long tubular bones / A.A. Belyaeva, N.E. Mach-son, E.Sh. Savadyan // Vestn. hir. -1987. - No. 10. - P.70 $-74$.

2. The use of high-energy lasers in the treatment of experimental acute hematogenous osteomyelitis / V.A. Privalov, O.S. Kushakovsky, A.V. Lappa et al. // New technologies in surgery: Tr. 3 Ross. scientific-practical conf. - Ufa, 1998 .-S. 64-66.

3. Privalov, V.A. Osteoperforation with a diode laser in the treatment of acute and chronic osteomyelitis / V.A. Privalov, I.V. Krochek, A.V. Lappa // Bull. VSNTS SB RAMS. - 2001. - Vol. 1, No. 3 (17). - S. 115-121.

4. Laser osteoperforation in the treatment of osteomyelitis / I.V. Krochek, V.A. Privalov, A.V. Lappa et al. / Abstracts of the 8th International Congress of the European Medical Laser Association (EMLA) and the 1st Russian Congress of the Medical Laser Association (RMLA). - M., 2001 .-- p. 111.

5. Sonis A.G. The results of the use of gravitational therapy in the treatment of patients with osteomyelitis of the lower extremities // Bulletin of Experimental and
Clinical Surgery. - 2010. - T 3, No. 4. - P. 377384

6. Khasanov A.G. The results of the use of plasma flows in the complex treatment of chronic osteomyelitis // Bulletin of experimental and clinical surgery. - T 3, No. 3. - 2010. - S. 210-214.

7. Radaev S.V. The use of nitrogen monoxide in the complex treatment of chronic osteomyelitis: author. dis. ... Cand. Samara, 2009 --- 136 p.

8. Alekseev D.G. Chronic osteomyelitis: features of complex treatment at the present stage / D.G. Alekseev, I. V. Ishutov, V.E. Batakov // Young Scientists for Healthcare of the Region: mater. scientificpractical conf. - Saratov, 2005 --- S. 237-238. 